\title{
Research on the UI and UE Design Techniques and the Status in HTML5 Website Development
}

\author{
Li Wang \\ Beijing Institude of Economic Management, \\ Beijing 100029,China
}

\begin{abstract}
In this paper, we conduct research on the UI and UE design techniques and the status in the HTML5 website development. With the continuous development of science and technology, the user demand for computer software design level enhances unceasingly, the software development workers must deep going research analysis of the target group, to truly understand the user groups like what kind of software, need what kind of software. It is human's behavior and cognition of the operational system affect design, management and improvement of related attributes, and studies the properties and the operation system and the process of interaction. Under this historical period of the time, we propose the novel perspective of the issues that is meaningful.
\end{abstract}

Keywords-UI and UE, Design Techniques, Status, HTML5, Website Development.

\section{Introduction}

With the rapid development of WEB technology and the improvement of people's life quality, the website has become an indispensable people daily life factors, which not only reflected in the WEB site is a large number of information sources in many areas, also in website provides many practical service for its users, such as online shopping, maps, weather forecast, and electronic library and so on. Whether information websites, or the application class web site, has adopted the latest dynamic web technology to design, dynamic web pages that can be according to the different situation to make the dynamic response, so it can provide a better user experience, such as the information update, content changing and style of the freedom of choice and so on, but these dynamic changes was not the website developers to complete, because it is impossible for most of the sites are equipped with a professional development team long-term maintenance. In the face of rapidly changing demand, caused a lot of, such as the front-end coding standards, front-end performance optimization such as front-end security holes to prevent problems. In order to solve these problems, a kind of advanced development idea and good design patterns the front end of the modular technology is gradually mature, and favored by the more and more companies and institutes [1-3].

According to the literature review, the popularly adopted web development tools could be generally summarized as follows. FrontPage. FrontPage is Microsoft has launched a powerful creation and management of the Web site of the program, to use it without programming can create rich in content and professional Web site. FrontPage through the gallery component provides definitions to add basic pictures, picture layout, add text to the images, rearrange the pictures, change the image size, make a miniature diagram, such as functions also has provided the drawing tool and simple image processing functions. (2) Visual InterDev. Visual InterDev was developed by the Microsoft to create and manage Web site integration tool software, Microsoft Visual Studio is the integration in the professional Web application development tools. (3) Dreamweaver. Dreamweaver is Macromedia's edit Web tools for the dynamic Web pages to the Web application development environment with the visual creative environment that helps users 
quickly in the development of Web applications [4-5].

With 3G and even 4G/LTE commercially and large-scale intelligent mobile terminal applications, mobile Internet is in rapid development. As leading role of the mobile Internet, mobile applications for end users, network operators and application developers, platform providers, investors will have a huge impact, is a major force to promote the development of future mobile Internet. HTML language is the most widely used current web programming languages, has many characteristics, such as easy to use, fast and browse across platforms. It not only made up for the inadequacy of the generation of the HTML, basic page effect with Flash, deployment headaches, so in the web page design, the adaptive updating has obvious advantages. Of the development process is basically standing in the browser on the shoulder, with convenient cross-platform transplantation, all resolution on the client browser, and thoroughly solves the problem to differences in equipment development, and support the API, CSS3 and programming logic. The following figure one illustrates the corresponding applications.

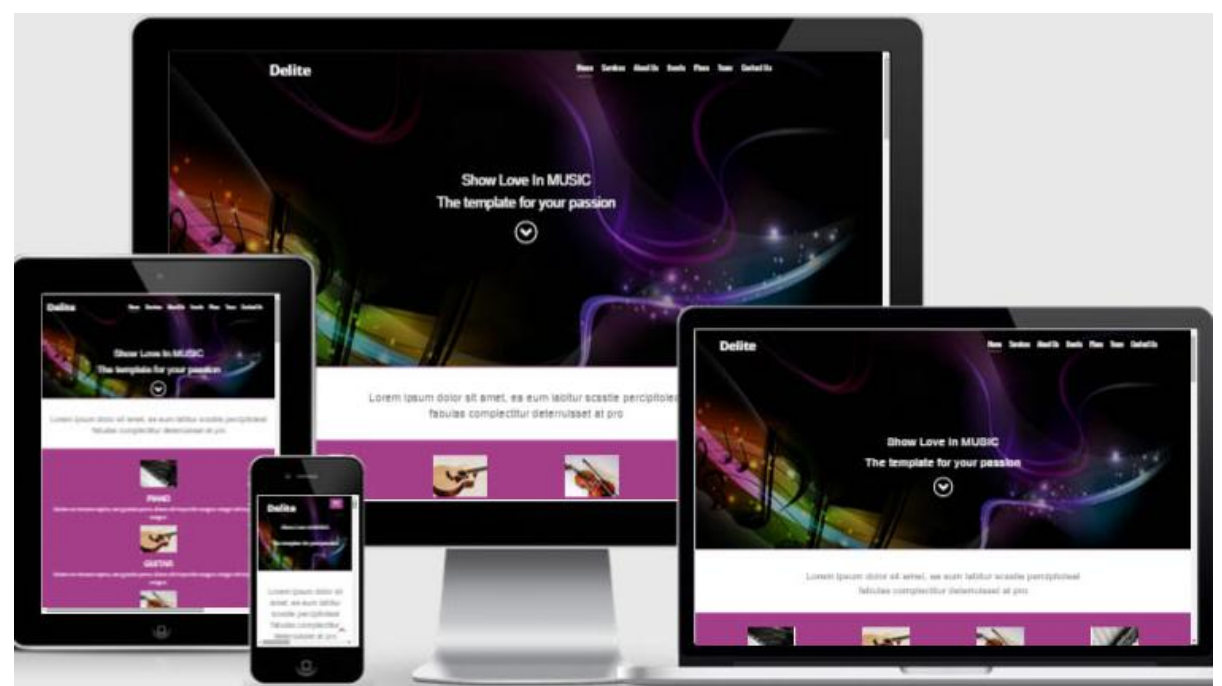

Figure 1. The Demonstration of the HTML5 based Cross-Platform Applications

In this paper, we conduct research on the UI and the UE design techniques and the status in HTML5 software development. In the following sub-sections, we will analyze the concepts with explanation.

\section{The Proposed Methodology}

The User Experience Principles. User experience originally refers to the users in the use of products or to enjoy the service was set up in the process of psychological feeling, involved with the product, system or service all aspects in the process of interaction. User experience research this field was set up to comprehensive analysis and the perspective of the user in contact with a product, system or the service or use feeling. The research is focused on valence and perceived value of a product or service delivery, and not only is the product or the performance of the system itself [6].

Behavior operation is combined with cognitive and social psychology theory to research the new methods of operation management. It is human's behavior and cognition of the operational system affect the design, management and improvement of related attributes, and studies the properties and the operation system and the process of interaction. Web page belongs to the man-machine interface 
design in the design of software interface type. To study from the perspective of people learned from e-commerce site user experience, and the people as the starting point of web site design can make the functionality of the site distribution, color collocation better adapt to and satisfy human physiological and psychological needs so that the e-commerce site more human, fit the user's requirements that can be organized as the follows. (1) Optimization of the web page content. It includes two aspects, on the one hand, content to be high quality, appropriate updates as optimization of website content, on the other hand, make it suitable for search engines to retrieve. (2) Website structure optimization as is the web page of storage as well as the relationship between internal links and reasonable adjustment, to reduce the basic page directory depth and important link of the page depth. Structural optimization is divided into physical structure and the logic structure optimization. (3) From raising the website user experience point of view, Web content mining task is how to improve the quality of information and help the user to filter the information is not interested and also can be used for mining user browsing preferences, to provide basic users with site information push and prediction, etc. (4) Compared with website optimization design and research in user experience optimization in terms of Web mining, less to the user experience optimization evaluation discussion. Look from evaluation point, we can be divided into focus on content and focus on user behavior evaluation.

The Principles of UI Design. Main interface of a web site is the first step to attract new users. Normally, the style of website main interface represents the team or individual style, users can easily by browsing the web site of the first to judge whether the style accepted for himself and for the next move. Google, for the example, this has been recognized as the world's largest search engine and its purpose is to provide people with free and easy information search service [7].

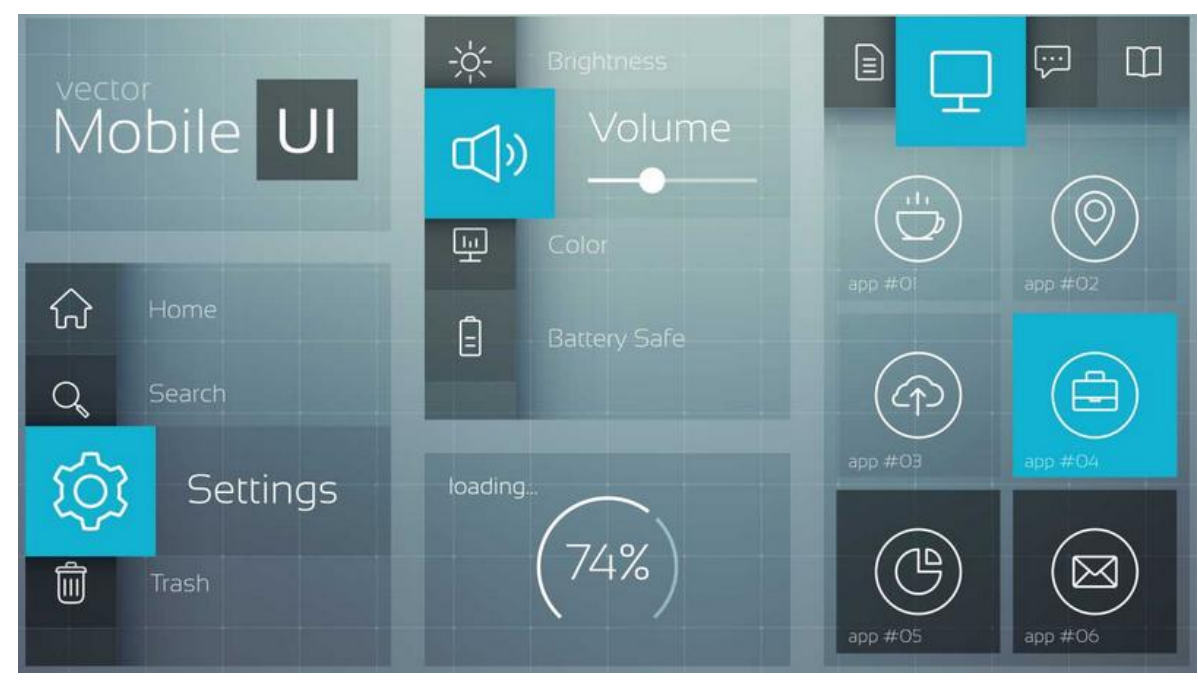

Figure 2. The Sample Enhance User Interface Demonstration

Web page has become the most typical, which is the most popular of human-computer interaction platform, web page design is the typical application of human-computer interface design. The whole process of web page design, through the "people-oriented" design idea, mainly involves the following aspects of content and unique perspectives.

- The use of images. In the early days of the web site, the picture is less, because of the relatively large file may affect the image display quality and now with the 
invention of the broadband network and application, we can design in web design bold use larger figure.

- Illustrations show. Illustration is an important performance in general web interface elements, although it is give priority to in order to beautify and auxiliary information, but its existence is not dispensable. On the one hand, it can help people understand and know the color, on the other hand can build relaxed and pleasant reading environment, build the affinity of the page.

- Icon. Icon is a graphic with characteristics of identity with reference as it is not only a kind of graphics, but also a logo while it is highly concentrated and quickly conveys information, the characteristics of easy to remember. Icon between content and adornment, the ICONS can be understood as a kind of adornment, because it is a lot of time in order to enhance the meaning.

- Motion graphics. On the Internet, motion graphics for challenging dynamic movement and sound effects, the range of motion graphics used in the site is also growing. Site in the use of the motion graphics, as not only can improve the efficiency of the user, and gives users in use process to experience the fun of operation, inspire their emotional experience.

In terms of the web interface design should also consider is the use of both convenience and visual effects. First of all, the site each page should maintain consistency, cannot bring confusion to the user; In terms of use, as far as possible to follow the rule, which allows users to easily adapt to. In interface design, it is important to stand in the user's standpoint. Can not only bring convenience to users, and visual impact, the web interface is each page designer should think about and pursuit. A don't consider for the user interface, no matter how good the site has a content, the good content are worthless things. Both practicality and the convenience of interface as of the course important, but can arouse the user's curiosity and interest, the user site, impressive interface is also very important.

The HTML5 Properties. HTML $5 \mathrm{draft}$, formerly known as general WebApplications1.0, by the WHATWG proposed in 2004, 2007 by the W3C, and established the new HTML working group. In September 2010 was recommended to the public the first formal draft. It is Web standards the huge leap in nearly a decade. Unlike previous versions, it is not only used to represent Web content that its mission is to bring the Web into a mature application platform, on the platform, video, audio, image, animation, and interactions with the computer have been standardized. A new generation of HTML 5 technology quickly became popular in the browser application software and the network management software applications brought new development well solves the shortcomings of the former. In the following list, we demonstrate the new properties of the HTML5.

- Data storage and offline Web applications. Local Storage can be permanent data, that is, for supporting the Local store browser will be specified in the user's Local allocate space for the permanent Storage of data, after the user closes the browser, open a browser can also obtain the data stored next time, unless the user take the initiative to delete the data [8].

- Multi-threaded support. After the corresponding task at the end of the background processing, the front desk as long as you use the callback function to receive processing the data returned. In addition to pay attention to the Worker can't operate DOM background and if we want to processing the daemon can change the DOM.

- Web Socket. Web Socket is a very important new features in HTML 5 specification, it can allow the existence of 
the two-way connection between the server and the client, the browser, the connection is real-time, data can be timely delivery, and the connection remains open until explicitly close it with the general conditions.

The Novel Website Development Paradigm. Application framework template website for its easy to develop and maintain the superiority of the Internet and the electronic business competition is more and more show its advantages. Application of MVC pattern, using the tag library technology, the use of language is a good way to implement frame templates and so that it can be easily applied to your site framework of template, which can improve the development efficiency and maintainability of the website and implement the function of page display and separation, easy to realize the unity of the style of the website. Front modular development and the basic idea of modular program design, namely on the Web based on the basic unit module and organize information, separate Web content, forming a number of relatively independent modules. Module is used to store the basic page elements of the container, and common page elements also made module, facilitate the call and management of these elements on the page. As a result, the front-end modular technology without the complex logic operations, but focuses on as the presentation layer as a skeleton of HTML and CSS, and JavaScript implementation behavior in the combined and code reuse of the three aspects, in order to reduce the circular dependencies between them, to reduce the coupling and improve design efficiency.

Web site home page design in web design, to maintain the continuity of the various pages, using block design and design of the Head as HTM as the Head of every web page, Design Foot. HTM as the footer of a web page with the head to striking, the color of the footer can highlight the key color to give priority to. Middle part content is often as the base colors with white, can put content is distinct. Intermediate business layer is responsible for handling user input, or to save the information sent to the data access layer that also can call the function in the data access layer again read these data. The modern dynamic website development technology available varied, all kinds of development pattern is being developed, development efficiency is becoming more and more high, and the development difficulty is also gradually decline. The only way for the development of modern web site is also the most efficient way is template development path. PHP technology, for example, a PHP website rapid development can be the reference template type DEDE, PHPCMS, Discuz template as they all have in common is encapsulates scenes, as saved the background development time which greatly reduces the technical difficulties and development costs.

\section{Conclusion}

In this paper, we conduct research on the UI and UE design techniques and the status in the HTML5 website development. It is well known that each web site interface image will largely affect different users on the site like we will be different for each person a different image of the impression, that appearance if good impression to the person, then can let a person to embrace all of them. Therefore we design the web interface, not only should have a generous and beautiful appearance that also must stand at the point of view of computer users. Under this guidance, in this paper, we propose the novel

UI and UE design techniques and the status in the HTML5 website development with the integration of the literature reviews that is meaningful.

\section{References}

[1] Song, Yujie, and Sanxing Cao. "HTML5-based tourism needs of users broadcast audio APP Design and Construction." (2015). 
[2] Pramadharma, Harestu, and Rung-Ching Chen. "Building Browser Extension to Develop Website Personalization Based on Adaptive Hypermedia System." Current Approaches in Applied Artificial Intelligence. Springer International Publishing, 2015. 316-325.

[3] Bryan, Howard, and Mark Anderson. "Accessibility Issues in HTML5: A comparison of HTML5 websites and those coded in earlier versions." International Journal on Recent and Innovation Trends in Computing and Communication 2.11 (2014): 3415-3421.

[4] Sowell, Eric. "Native APIs, HTML5, and CSS3 on Mobile Today." Mobile ASP. NET MVC 5. Apress, 2013. 157-179.
[5] Seipp, Karsten, and Kate Devlin. "The one hand wonder: a framework for enhancing one-handed website operation on touchscreen smartphones." (2014): 5-13.

[6] Savel, Craig, et al. "Web-based, mobile-device friendly, self-report survey system incorporating avatars and gaming console techniques." Online journal of public health informatics 6.2 (2014).

[7] Mehri, Seyedeh Marjaneh Ahmadi. Effect of packet loss on VP8 encoded videos using HTML5 based streaming. Diss. Blekinge Institute of Technology, 2014.

[8] Pastore, Serena. "The role of open web standards for website development adhering to the one web vision." International Journal of Engineering and Technology 2.11 (2012): 1824-1834. 